\title{
Mecp2 Deletion from Cholinergic Neurons Selectively Impairs Recognition Memory and Disrupts Cholinergic Modulation of the Perirhinal Cortex
}

\author{
Elizabeth C. Ballinger, ${ }^{1,2,3}$ Christian P. Schaaf, ${ }^{7,8,11}$ Akash J. Patel, ${ }^{7,9}$ Antonia de Maio, ${ }^{6,7,8}$ Huifang \\ Tao, ${ }^{7,8}$ DDavid A. Talmage, ${ }^{1,4,5}{ }^{-}$Huda Y. Zoghbi, ${ }^{6,7,8,10}$ and ${ }^{\circledR}$ Lorna W. Role ${ }^{1,4}$
}

https://doi.org/10.1523/ENEURO.0134-19.2019

\begin{abstract}
${ }^{1}$ Department of Neurobiology and Behavior, Stony Brook University, Stony Brook, New York 11794, ${ }^{2}$ Program in Neuroscience, Stony Brook University, Stony Brook, New York 11794, ${ }^{3}$ Medical Scientist Training Program, Stony Brook University, Stony Brook, New York $11794,{ }^{4}$ Center for Nervous System Disorders, Stony Brook University, Stony Brook, New York 11794, ${ }^{5}$ Department of Pharmacological Sciences, Stony Brook University, Stony Brook, New York 11794, ${ }^{6}$ Program in Developmental Biology, Baylor College of Medicine, Houston, Texas $77030,{ }^{7}$ Jan and Dan Duncan Neurological Research Institute at Texas Children's Hospital, Baylor College of Medicine, Houston, Texas 77030, ${ }^{8}$ Department of Molecular and Human Genetics, Baylor College of Medicine, Houston, Texas 77030 , ${ }^{9}$ Department of Neurosurgery, Baylor College of Medicine, Houston, Texas $77030,{ }^{10}$ Howard Hughes Medical Institute, Baylor College of Medicine, Houston, Texas 77030, and ${ }^{11}$ Institute of Human Genetics, Heidelberg University, 69120 Heidelberg, Germany
\end{abstract}

\begin{abstract}
Rett Syndrome is a neurological disorder caused by mutations in the gene encoding methyl CpG binding protein 2 (MeCP2) and characterized by severe intellectual disability. The cholinergic system is a critical modulator of cognitive ability and is affected in patients with Rett Syndrome. To better understand the importance of MeCP2 function in cholinergic neurons, we studied the effect of selective Mecp2 deletion from cholinergic neurons in mice. Mice with Mecp2 deletion from cholinergic neurons were selectively impaired in assays of recognition memory, a cognitive task largely mediated by the perirhinal cortex $(\mathrm{PRH})$. Deletion of Mecp2 from cholinergic neurons resulted in profound alterations in baseline firing of L5/6 neurons and eliminated the responses of these neurons to optogenetic stimulation of cholinergic input to $\mathrm{PRH}$. Both the behavioral and the electrophysiological deficits of cholinergic Mecp2 deletion were rescued by inhibiting ACh breakdown with donepezil treatment.
\end{abstract}

Key words: acetylcholine; Mecp2; perirhinal; recognition; Rett Syndrome

\section{Significance Statement}

Rett Syndrome, a developmental disorder characterized by multiple deficits including intellectual disability, is caused by mutations in the MECP2 gene. In this study, Mecp2 was selectively deleted from cholinergic neurons in mice causing a specific impairment of recognition memory that was reversed following chronic administration of the acetylcholinesterase inhibitor donepezil. As recognition memory engages the perirhinal cortex, we examined the effects of Mecp2 deletion from cholinergic neurons on the physiology of perirhinal cortical neurons and found a reduction in the variability of baseline firing and impaired responsiveness to optogenetic stimulation of cholinergic input. Our findings are consistent with a loss of encoding capacity in the perirhinal cortex and suggest a possible electrophysiological substrate for the altered profile of recognition memory performance. 


\section{Introduction}

Rett Syndrome is a childhood neurologic disorder that affects 1 in 10,000 girls and is caused by mutations in a gene known as MECP2 (Rett, 1966; Hagberg et al., 1983; Lewis et al., 1992; Amir et al., 1999; Neul et al., 2010). The phenotype is complex and includes intellectual disability, breathing disturbances when awake, seizures, autonomic dysfunction, autistic features, stereotypies, locomotor defects, and gastrointestinal dysfunction. MECP2 encodes methyl CpG binding protein 2, a transcriptional regulator whose loss leads to both decreases and increases in gene expression (Chahrour et al., 2008). Discerning what cell types and Mecp2 target genes contribute to which aspects of the Rett Syndrome phenotype has posed a significant challenge. This challenge has been addressed by selectively removing Mecp2 from distinct neuronal populations, revealing which neuronal populations and brain regions contribute to the key features of the disorder. Genetically targeted approaches have previously been applied to the GABAergic, glutamatergic, Sim1-expressing, and aminergic systems; and have provided an added level of resolution in the investigation of the significance of these neurotransmitter systems to Rett Syndrome (Fyffe et al., 2008; Samaco et al., 2009; Chao et al., 2010; Meng et al., 2016).

Given that a key characteristic of individuals with Rett Syndrome is severe cognitive impairment (Rett, 1966; Hagberg et al., 1983; Neul et al., 2010) and the well established role of the cholinergic system in cognitive functions (for review, see Ballinger et al., 2016), we have used the Mecp2 deletion approach to assess the role of MeCP2 in cholinergic neurons and the resulting phenotypes due to its loss. Prior studies in both humans and animal models have implicated alterations in acetylcholine (ACh) signaling in Rett Syndrome. Postmortem immunohistochemical studies of the brain from individuals with Rett Syndrome have shown profound cholinergic deficits: there are reduced numbers of choline acetyltransferase (ChAT)-positive cells in the basal forebrain, reduced ChAT

Received April 4, 2019; accepted September 17, 2019; First published September 27, 2019

The authors declare no competing financial interests.

Author contributions: E.C.B., C.P.S., D.A.T., H.Y.Z., and L.W.R. designed research; E.C.B., C.P.S., A.J.P., A.d.M., and H.T. performed research; E.C.B. and C.P.S. analyzed data; E.C.B., C.P.S., D.A.T., H.Y.Z., and L.W.R. wrote the paper.

This work was supported by the following awards: National Institutes of Health (NIH) Grant MH-105087-02 (to E.C.B.); NIH Grant NS-057819 (to H.Y.Z.); NIH Grant NS-022061 (to L.W.R.); International Rett Syndrome Foundation ANGEL Grant (to H.Y.Z.); and the Rett Syndrome Research Trust (to H.Y.Z.).

L. W. Role's present address: Office of the Scientific Director, National Institute of Neurological Disorders and Stroke, National Institutes of Health, Bethesda, MD 20892.

Acknowledgments: We thank Saira Khan, Jonathan McGuire, Andrew Walcott, and Paul Yarabe for contributions to this work.

Correspondence should be addressed to Elizabeth C. Ballinger at Elizabeth.Ballinger@StonyBrookMedicine.edu or Christian P. Schaaf at Schaaf@bcm.edu.

https://doi.org/10.1523/ENEURO.0134-19.2019

Copyright (C) 2019 Ballinger et al.

This is an open-access article distributed under the terms of the Creative Commons Attribution 4.0 International license, which permits unrestricted use, distribution and reproduction in any medium provided that the original work is properly attributed. and VAChT activity, and reduced cholinergic receptor expression (Kitt et al., 1990; Wenk and Mobley, 1996; Wenk, 1997; Wenk and Hauss-Wegrzyniak, 1999; Yasui et al., 2011). Likewise, mice lacking MeCP2 have shown reductions in both $\mathrm{ACh}$ and $\mathrm{ChAT}$, dramatically attenuated cholinergic currents in electrophysiological experiments, and altered cholinergic receptor expression profiles (Ward et al., 2009; Ricceri et al., 2011; Oginsky et al., 2014; but see also Zhou et al., 2017). Finally, cholinergic marker reductions as evaluated by SPECT imaging in vivo have been correlated with clinical severity in patients with Rett Syndrome (Brašić et al., 2012).

To evaluate the potential contribution of the cholinergic system to phenotypes observed in Rett Syndrome in a systematic way, we have used a Cre-Lox system to selectively delete Mecp2 from cholinergic neurons only (see Fig. 2A). We evaluated the performance of these mice on a number of cognitive tasks and found specific deficits in novel object recognition-behaviors that depend on intact functioning of the perirhinal cortex $(\mathrm{PRH}$; for review, see Dere et al., 2007). We then explored the electrophysiological and molecular mechanisms underlying specific cognitive deficits in novel object recognition.

\section{Materials and Methods}

\section{Animals}

For electrophysiological, behavioral, and molecular experiments transgenic male mice expressing Cre recombinase under control of the Chat promoter (Chat-Cre; stock \#006410, The Jackson Laboratory; RRID:IMSR_JAX: 006410) maintained on a C57 background were crossed with female mice heterozygous for a floxed Mecp2 allele (Mecp2 flox; stock \#007177, The Jackson Laboratory; RRID:IMSR_JAX:007177) maintained on a 129 background. This cross generated the following four different genotypes of male offspring: mice with no transgenes, mice with the Chat-Cre transgene only, mice with the Mecp2 flox allele only, and mice with both transgenes (Chat-Mecp2 $2^{-1 y}$; see Fig. 2A). Male mice from the F1 generations of the original cross were used for experiments. Mice of all genotypes were born at the expected Mendelian ratios and were healthy appearing at birth. However, Chat-Mecp2-/y mice did exhibit a phenotype of reduced survival, with most Chat-Mecp2-/y mice dying between 16 and 36 weeks of age while all other genotypes lived for $40+$ weeks. Chat-Mecp2 $2^{-/ y}$ mice gained weight at rates similar to those of genetic controls and were generally healthy appearing until death, which was an acute/subacute event of unknown cause.

All mice were maintained on a $12 \mathrm{~h}$ light/dark cycle and allowed food and water ad libitum. Mice were either pair or group housed. No singly housed mice were used for behavioral experiments. The same cohort of mice was examined on both the partition test and the novel object recognition test. A separate cohort of mice was examined on conditioned fear testing. A subset of this second cohort also underwent Morris water maze testing. A third cohort of mice was used for electrophysiological experiments. 


\section{Context-conditioned fear and cue-conditioned fear}

Fear conditioning was performed as previously described (Takeuchi et al., 2011). This test was conducted when mice were 21 weeks old. Each mouse was placed in a sound-attenuated chamber and allowed to explore freely for 2 min. An $80 \mathrm{~dB}$ white noise, the conditioned stimulus (CS), was presented for $30 \mathrm{~s}$; this was followed by a mild $(2 \mathrm{~s}, 1 \mathrm{~mA})$ footshock, the unconditioned stimulus (US). Two more CS-US pairings were presented with 2 min interstimulus intervals (ISIs). Context testing was conducted $1 \mathrm{~d}$ after conditioning in the same chamber. Cued testing with altered context was conducted on the same day, following the context testing, using a triangular box made of white opaque Plexiglas and vanilla scent presented behind the separation to change olfactory stimulus. Data acquisition, control of stimuli (i.e., tones and shocks), and data analysis were performed automatically using the Actimetrics FreezeFrame3 System (Coulbourn Instruments; RRID:SCR_014429). For context-conditioned fear, the percentage of time spent freezing in the conditioned context on testing day was calculated and compared between groups. For cue-conditioned fear, on testing day the cue-specific time spent freezing was calculated as follows: cue-specific freezing $=(\%$ time freezing during cue $)-(\%$ time freezing before cue). Cue-specific freezing was then compared between groups.

\section{Morris water maze}

Morris water maze was performed as previously described (Takeuchi et al., 2011). This test was conducted when mice were 17 weeks old. A circular pool $(120 \mathrm{~cm}$ in diameter) was filled with water $\left(21 \pm 1^{\circ} \mathrm{C}\right)$, in which nontoxic white tempera paint was mixed to make the surface opaque. For the invisible platform test, a white-colored platform was placed at the center in one of four quadrants of the pool (southwest area) and submerged $1 \mathrm{~cm}$ below the water surface so that it was invisible at water level. The location of the platform was fixed at the same quadrant, while the start position of swimming was varied. Mice were given eight trials per day (two blocks of four trials each) for 4 consecutive days, during which they were allowed $60 \mathrm{~s}$ to find the platform. Each trial was separated by an intertrial interval of 1-2 min, with each block separated by an interblock interval of at least $1 \mathrm{~h}$. Once the mouse located the platform, it was permitted to stay on it for $10 \mathrm{~s}$. If the mouse did not find the platform within $60 \mathrm{~s}$, it was guided to the platform and placed on it for $20 \mathrm{~s}$. To evaluate the spatial reference memory, all mice were given a probe trial following the training, at least $1 \mathrm{~h}$ after their last training trial on day 4 . The probe trial consisted of removing the platform from the pool and allowing the mice to swim for $60 \mathrm{~s}$ in their search. A record was kept of the swimming time (in seconds) in the pool quadrant where the platform had previously been placed. During the visible platform test, a colored platform was placed in the quadrant $1 \mathrm{~cm}$ above the surface of the water, and its location was always varied randomly in each trial. Swim speed (in centimeters per second), latency time to find the platform (in seconds), and the time that each mouse swam in the target quadrant were re- corded by video camera and analyzed by a computercontrolled video-tracking system (Ethovision XT, Noldus Information Technology; RRID:SCR_000441).

\section{Partition test}

The partition test of social interaction was conducted when mice were 14 weeks old. Test subjects were individually housed in a standard cage divided by a perforated partition, as described previously (Moretti et al., 2005). Male partner mice of the same age were placed into the side opposite the test subject at least $18 \mathrm{~h}$ before testing social interaction. Recording of social interest (i.e., the amount of time test subjects spent at the partition actively interested in partner mice) was performed as described previously (Moretti et al., 2005).

\section{Recognition memory training}

Recognition memory testing was conducted when mice were 20 weeks old. The behavioral apparatus consisted of two empty rodent cages cleaned with $40-50 \% \mathrm{EtOH}$, as follows: one cage was used as a habituation arena, and the other cage was used as the test arena. On each day for 5 consecutive days, mice were placed in the habituation arena for 5 min and then transferred to the test arena for $5 \mathrm{~min}$. On days 1,2 , and 3 , the test arena contained the same pair of two identical objects (Lego objects, $\sim 12 \mathrm{~cm}$ high). On day 4 , one of the objects was replaced with a novel object (Lego object of same height, but of different color pattern and shape than the familiar object). On day 5 , the test arena again contained the pair of identical familiar objects. The amount of time spent exploring each object was quantified during each session. The following behaviors were considered "exploration": whisking the object, biting the object, touching the object, and nose oriented toward and within $2 \mathrm{~cm}$ of the object.

\section{Viral injection}

Before the electrophysiological recording, a subset of mice underwent viral injection to facilitate optogenetic stimulation of cholinergic neurons. To target cholinergic neurons, we used a Cre-dependent virus, and the experiments were limited to Chat-Cre and Chat-Mecp2 ${ }^{-1 y}$ mice. Mice for optogenetic experiments were anesthetized with isoflurane at 11 weeks of age and mounted on a stereotaxic frame (Kopf Instruments) with a heated stage. An incision in the scalp was made, and a small hole was drilled in the skull above the left nucleus basalis magnocellularis [NBM; coordinates from bregma: anteroposterior $(\mathrm{AP}),-0.7 \mathrm{~mm}$; mediolateral $(\mathrm{ML}), 1.7 \mathrm{~mm}$; $z$-axis, $-4.0 \mathrm{~mm}$. A total of $0.5 \mu \mathrm{l}$ of either AAV9-Ef1a-DIOChETA-eYFP or AAV9-CAG-DIO-oChIEF-tdTomato was injected using a microsyringe (Hamilton). Mice were used for electrophysiological recording 3 weeks after infection.

\section{Electrophysiological recording}

For electrophysiological experiments, mice that were at least 13 weeks of age were anesthetized with isoflurane and placed on a surgical stereotax (Kopf Instruments) with a heated stage. A craniotomy over the left perirhinal cortex was performed and a tungsten electrode of either 1 or $5 \mathrm{M} \Omega$ (A-M Systems) was positioned into the posterior 
$\mathrm{PRH}$ (coordinates from bregma: AP, $-3.25 \mathrm{~mm}$; $z$-axis, -3.35 to $-3.85 \mathrm{~mm}$; $\mathrm{ML}$ from temporal ridge, -200 to $+500 \mu \mathrm{m})$. Extracellular recordings were preamplified by the head stage of A-M Systems amplifier. For optogenetic experiments, mice (i.e., Chat Cre mice with or without Mecp2 flox) received an additional craniotomy over the left NBM through which a $1 \mathrm{M} \Omega$ parylene-C-insulated tungsten electrode (A-M Systems) and a $200 \mu \mathrm{m}$ optical fiber (Thorlabs) coupled to a $473 \mathrm{~nm}$ laser (Shanghai Dream Lasers Technology) were positioned in the NBM.

Signals were acquired at a sampling rate of $40 \mathrm{kHz}$ and bandpass filtered at $100-1000 \mathrm{~Hz}$ by the amplifier (A-M Systems) before being passed through a Humbug Noise Eliminator (A-M Systems) and then displayed on a Tektronix TDS 2014B oscilloscope and fed to a Cambridge Electronic Design 1401 data board for visualization and collection using Spike 2 software (Cambridge Electronic Design). Laser stimuli used for optical stimulation of cholinergic neurons consisted of 20 laser pulses of $1 \mathrm{~ms}$ duration delivered at a frequency of $10 \mathrm{~Hz}$.

\section{Relocalization of recording site}

At the end of each recording session, an electrolytic lesion was created by passing $100 \mu \mathrm{A}$ of current for $45 \mathrm{~s}$ through the recording electrode to facilitate relocalization of the recording site. The mouse was then perfused transcardially and brain slices were obtained as discussed below.

For a subset of mice, slices containing the perirhinal cortex were stained using NeuroTrace (Thermo Fisher Scientific) blue fluorescent Nissl stain. The perirhinal cortex was defined histologically as per Beaudin et al. (2013). In short: the medial border was defined by the external capsule, the dorsal border was distinguished by the loss of the prominent layer IV seen in the dorsally adjacent temporal association cortex, the ventral border was distinguished by loss of the prominent layer II seen in the ventrally adjacent entorhinal cortex.

For all mice, slices containing the $\mathrm{PRH}$ were imaged on a stereoscope (Zeiss). For mice used for optogenetic experiments, slices containing the NBM were imaged to confirm viral expression.

\section{Sample preparation for light and confocal microscopy}

Mice were anesthetized with a 9:1 mixture of ketamine and xylazine and transcardially perfused with $1 \times$ PBS followed by $4 \%$ PFA. Brains were removed and postfixed overnight in 4\% PFA before being sucrose equilibrated and frozen in OCT (optimal cutting temperature) compound. Brains were then cryosectioned (Leica Biosystems) at $50 \mu \mathrm{m}$ thickness.

\section{Immunohistochemistry}

For Nissl staining brain slices were blocked and permeabilized for $30 \mathrm{~min}$ in $1 \times$ PBS with $5 \%$ donkey serum and $0.1 \%$ Triton $\mathrm{X}-100$, and then incubated for $90 \mathrm{~min}$ in a 1:200 dilution (in blocking/permeabilization solution) of the NeuroTrace blue fluorescent Nissl stain (Thermo Fisher Scientific) followed by three 5 min washes in $1 \times$ PBS. Slices were mounted with DAPI Fluoromount-G
(Southern Biotech). All steps were performed at room temperature.

For MeCP2 and ChAT staining, brain slices were blocked and permeabilized for $1 \mathrm{~h}$ at room temperature in $1 \times$ PBS with $5 \%$ donkey serum and $0.5 \%$ Triton X-100 and then incubated for $48 \mathrm{~h}$ on a shaker at $4^{\circ} \mathrm{C}$ in $1: 200$ rabbit anti-MeCP2 (catalog \#3456, Cell Signaling Technology; RRID:AB_2143849) or 1:200 goat anti-ChAT (EMD Millipore; RRID:AB_2079751) diluted in blocking solution. Slices were then washed three times for $10 \mathrm{~min}$ each in $1 \times$ PBS and then incubated overnight in 1:500 Invitrogen goat anti-rabbit (Thermo Fisher Scientific) or 1:1000 Invitrogen donkey anti-goat (Thermo Fisher Scientific). After three 10 min washes in $1 \times$ PBS, slices were mounted with Vectashield (Vector Laboratories; RRID:AB_2336788) with DAPI and imaged on a confocal microscope.

\section{Electrophysiological data analysis}

Extracellular recordings were sorted off-line using the Offline Sorter (Plexon). Features of the waveforms were extracted, and individual units were demarcated by manually identifying clusters of waveforms in a twodimensional feature space of spike properties (Gray et al., 1995). The quality of each sort was rated according to the isolation distance between clusters within the recording. Only recordings of high sort quality, with $<5 \%$ overlap with other clusters, were used for further analysis. Units with firing rates $<0.05 \mathrm{~Hz}$ were excluded from further analysis.

The variability of the baseline firing rate was quantified by calculating the Fano factor (FF) of the firing rate computed for each $10 \mathrm{~s}$ bin during the $300 \mathrm{~s}$ preceding optical stimulation.

Responses to optogenetic stimulation were evaluated using a permutation test of the $F$ statistic with 10,000 permutations comparing interspike intervals occurring within the $140 \mathrm{~s}$ immediately before and after stimulation. To identify delayed responders, the same analysis was performed comparing the $140 \mathrm{~s}$ immediately before stimulation and a sliding $140 \mathrm{~s}$ window following stimulation. Each slide step was $70 \mathrm{~s}$. A response was detected if the resultant $p$ value from these permutation tests was $<0.05$. A unit was considered to exhibit an "early laser" response if a response was detected in the first $140 \mathrm{~s}$ window following stimulation. A unit was considered to exhibit a "delayed" response if a response was detected at any later window within the first $560 \mathrm{~s}$ following stimulation. If no responses were detected within the first $560 \mathrm{~s}$ following stimulation, the unit was considered to have no response.

\section{Donepezil pump implantation}

A subset of Chat-Mecp2 $2^{-/ y}$ mice generated using the same breeding strategy as described above underwent subcutaneous implantation of an osmotic minipump (model 2006, Alzet). Mice were anesthetized with isoflurane, and an incision was created over the left shoulder or caudal skull. Hemostats were lubricated with saline and then passed through the incision and used to open the subcutaneous space by separating the skin from the subcutaneous fascia. The pump was then implanted in this 
Table 1: Statistical methods by figure

Figure 2

$B$ : time to reach platform in Morris water maze: group $x$ testing session interaction

C: comparison by genotype of the percentage of time spent freezing to context

$D$ : comparison by genotype of the percentage of time spent freezing to cue

$E$ : comparison by genotype of time with novel/time with familiar on test day

$F$ : time spent at partition: group $\times$ testing session interaction

Figure 3

C: comparison by genotype of Fano factor of firing rate

$D$ : comparison by genotype of firing rate

Figure 5

C: within-unit comparison of variance of ISI prestimulation and poststimulation

$D$ : comparison by genotype of response rates

Figure 6

$A$ : comparison by treatment type of time with novel/time with familiar on test day

$B$ : comparison by treatment type of firing rate variability

$D$ : within unit comparison of variance of ISI prestimulation and poststimulation

$E$ : comparison by treatment type of response rates
Type of analysis

Results of analysis

Two-way repeated-measures ANOVA

Kruskal-Wallis test

$F_{(9,100)}=0.902$

$p=0.527$

Kruskal-Wallis test

$H_{(3)}=4.64$

$p=0.20$

Kruskal-Wallis test

$H_{(3)}=6.46$

$p=0.09$

$H_{(3)}=22.97$

$p<0.0005$

Two-way repeated-measures ANOVA

$F_{(6,118)}=4.908$

$p<0.0005$

Kruskal-Wallis test

$H_{(3)}=8.92$

$p=0.03$

Kruskal-Wallis test

$H_{(3)}=6.62$

$p=0.085$

Permutation test of $F$ statistic

$\chi^{2}$ test of homogeneity

$\mathrm{CR}(2)=6.02 \quad p=0.049$

Wilcoxon rank sum test

Rank sum $=120 \quad p=0.03$

Wilcoxon rank sum test

Rank sum $=293 \quad p=0.087$

Permutation test of $F$ statistic

$\chi^{2}$ test of homogeneity

$\mathrm{CR}(2)=4.15$

$p=0.126$ space, and the mouse was allowed to recover for 2 weeks. Pumps delivered either sterile saline or donepezil $\mathrm{HCl}$ (Biotang) in saline at a dose of $0.3 \mathrm{mg} / \mathrm{kg} / \mathrm{d}$.

\section{Statistics}

Performance on the partition test and Morris water maze was compared using a repeated-measures ANOVA design with genotype and testing session included as factors. Post hoc pairwise comparisons were performed using the Tukey's test. Performance on conditioned fear tests, baseline firing rate, firing rate variability, and performance on the test day of Novel Object Recognition for non-drug-treated mice were compared using the KruskalWallis test. Response rates to optogenetic stimulation were compared between genotypes using a $\chi^{2}$ test of homogeneity, the Cressie-Read Power-Divergence Statistic method, as this method is modestly superior to the traditional Pearson method for small tables (Rudas, 1986; Thorvaldsen et al., 2010). All other comparisons were performed with the Wilcoxon rank sum test. The repeated-measures ANOVA was performed in SPSS (IBM; RRID:SCR_002865). All other comparisons were performed in Matlab (MathWorks; RRID:SCR_001622). Details of the statistical analysis are summarized in Table 1.

\section{Results}

\section{Cognitive phenotyping of Chat-Mecp2 ${ }^{-/ y}$ mice}

As cholinergic signaling plays a vital role in mediating cognition (Ballinger et al., 2016) and intellectual disability is a central phenotype of MECP2 disorders, we first asked whether selective Mecp2 deletion from cholinergic neurons altered performance in several learning-related cognitive tasks. We used a Cre-lox system to selectively delete Mecp2 from cholinergic neurons (Figs. 1, 2A). This system has previously been shown to effectively reduce Mecp2 expression, as measured by immunofluorescence (Ito-Ishida et al., 2015; Zhang et al., 2016).

Chat-Mecp2-/y mice performed at control levels on the Morris water maze, an assay of spatial memory (Fig. $2 B ; n$ $=12$ for WT, Chat Cre, and Mecp2 flox mice; $n=11$ for Chat-Mecp2 $2^{-/ y}$ mice; repeated-measures ANOVA: interaction effect for genotype $\times$ testing day: Wilks' lambda $=$ 0.827, $\left.F_{(9,100)}=0.902, p=0.527\right)$. Similarly, Chat-
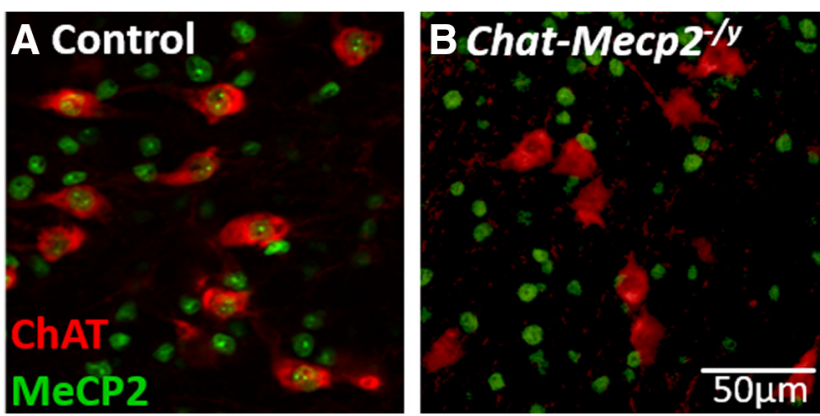

Figure 1. MeCP2 expression is eliminated in cholinergic neurons in Chat-Mecp2 $2^{-/ y}$ mice. $\boldsymbol{A}$, In a representative slice from the basal forebrain of a no transgene control mouse, MeCP2 expression (green) is apparent in the nuclei of cholinergic neurons (antiChAT stained, red). B, In a Chat-Mecp2 $2^{-/ y}$ mouse, however, MeCP2 expression in cholinergic neurons was not detected. 
A

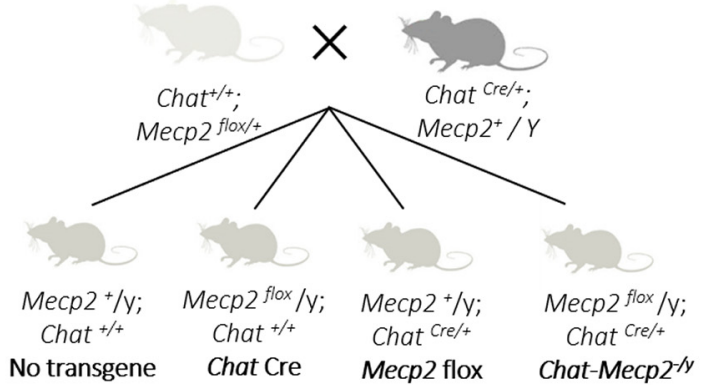

B

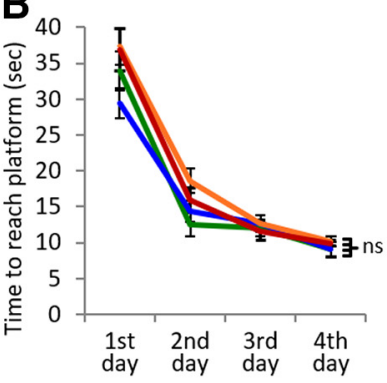

D Cue conditioned fear

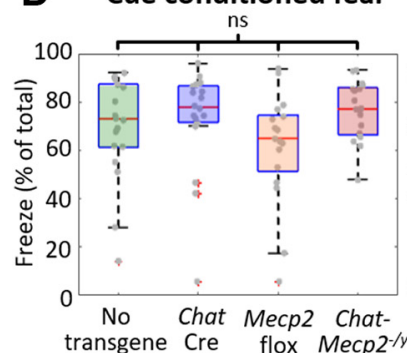

C Context conditioned fear

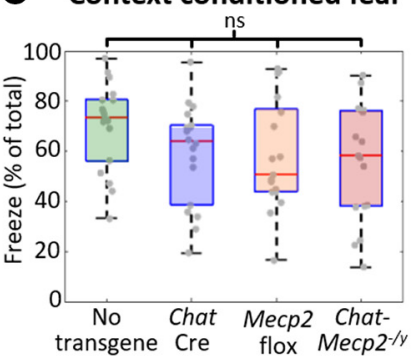

E Novel object recognition
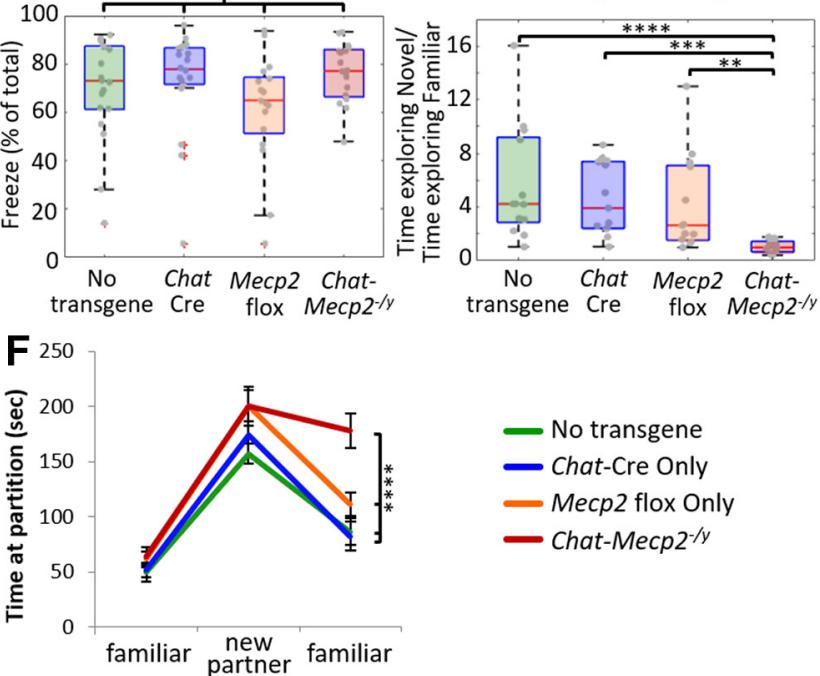

Figure 2. Mecp2 cholinergic selective knock-out mice are impaired in recognition memory of either an object or a conspecific. $\boldsymbol{A}$, Mecp2 flox mice were crossed with Chat Cre mice to generate Mecp2 selective knock-out mice (Chat-Mecp2-/y) and all three genetic controls. $\boldsymbol{A}-\boldsymbol{E}$, Chat-Mecp2 $2^{-/ y}$ mice $(\boldsymbol{A})$ performed at control levels on the Morris water maze $(\boldsymbol{B}$; repeated-measures ANOVA: interaction effect for genotype $\times$ testing day: Wilks' lambda $\left.=0.827, F_{(9,100)}=0.902, p=0.527\right)$, contextconditioned fear (C; Kruskal-Wallis test: $\left.H_{(3)}=4.64, p=0.20\right)$, and cue-conditioned fear (D; Kruskal-Wallis test: $H_{(3)}=6.46, p=$ 0.09). $\boldsymbol{E}$, However, Chat-Mecp2 $2^{-/ y}$ mice were impaired on novel object recognition (Kruskal-Wallis test: $H_{(3)}=22.97, p<$ 0.0005). Post hoc comparisons revealed that Chat-Mecp2 $2^{-/ y}$ mice were significantly different from all three genetic controls (no transgene, $p=0.0001$; Chat Cre, $p=0.0004$; Mecp2 flox, $p$ $=0.0079) . \boldsymbol{F}$, Chat-Mecp2 $2^{-/ y}$ mice showed reduced preference for the novel object introduced on day 4 (novel/familiar object ratio: mean, 1.038; SD, 0.48) than all three genetic controls (no transgene novel/familiar object ratio: mean, 5.6; SD, 4.30; Chat Cre novel/familiar object ratio: mean, 4.63; SD, 2.68; Mecp2 flox novel/familiar object ratio: mean, 4.18; SD, 3.62). Chat-Mecp2 $2^{-/ y}$
Figure 2. continued

mice were also impaired on the partition test (repeatedmeasures ANOVA: interaction effect for genotype $\times$ behavior session: Wilks' lambda $\left.=0.64 ; F_{(6,118)}=4.908 ; p<0.0005\right)$. Pairwise comparisons revealed that Chat-Mecp2 ${ }^{-l y}$ were significantly different from both no transgene mice $(p<0.0005)$ and Chat Cre mice $(p=0.001)$, although the difference between Chat-Mecp2 $2^{-/ y}$ and Mecp2 flox mice did not reach significance ( $p$ $=0.079$ ). On the partition test, Chat-Mecp2 ${ }^{-/ y}$ mice were impaired in their ability to recognize a familiar mouse and spent longer interacting with the familiar mouse on re-presentation (mean, $178.05 \mathrm{~s}$; SD, 63.02) than any of the genetic controls (no transgene: mean, $86.58 \mathrm{~s}$; SD, 47.92; Chat Cre: mean, $82.38 \mathrm{~s}$; SD, 51.89; Mecp2 flox: mean, 111.31s; SD, 43.97). Error bars represent the SEM. $* * p \leq 0.01, * * * p \leq 0.001, * * * * p \leq 0.0005$, ns $=$ non significant.

$\mathrm{Mecp2}^{-/ y}$ mice performed at control levels on the contextconditioned fear assay (Fig. 2C; $n=18$ for WT and Chat Cre mice; $n=17$ for Mecp2 flox and Chat-Mecp2 $2^{-/ y}$ mice; Kruskal-Wallis test, $H_{(3)}=4.64, p=0.20$ ) and cueconditioned fear assay (Fig. $2 D ; n=18$ for WT and Chat Cre mice; $n=17$ for Mecp2 flox and Chat-Mecp2 ${ }^{-/ y}$ mice; Kruskal-Wallis test, $H_{(3)}=6.46, p=0.09$ ).

Chat-Mecp2 ${ }^{-/ y}$ mice were impaired in recognition memory, as measured by the novel object recognition task (Fig. 2E; $n=13$ for no transgene, Chat Cre, and Mecp2 flox mice; $n=12$ for Chat-Mecp2 ${ }^{-/ y}$ mice; Kruskal-Wallis test, $\left.H_{(3)}=22.97, p<0.0005\right)$. Post hoc comparisons revealed that Chat-Mecp2 ${ }^{-/ y}$ mice showed significantly reduced preference for the novel object compared with all three genetic controls (no transgene, $p=0.0001$; Chat Cre, $p=0.0004$; Mecp2 flox; $p=0.0079$; no transgene novel/familiar object ratio: mean, 5.6; SD, 4.30; Chat Cre novel/familiar object ratio: mean, 4.63; SD, 2.68; Mecp2 flox novel/familiar object ratio: mean, 4.18; SD, 3.62). No other significant differences were found. Chat-Mecp2-/y mice also performed abnormally in the partition test assay of social interaction and memory (Fig. $2 F ; n=16$ /group; repeated-measures ANOVA: interaction effect for genotype $\times$ behavior session, Wilks' lambda $=0.64, F_{(6,118)}=$ 4.908, $p<0.0005)$. Although Chat-Mecp2 $2^{-/ y}$ mice originally interacted with the familiar mouse and the novel mouse at control levels, when the familiar mouse was reintroduced at the end of the trial, Chat-Mecp2- ${ }^{-/ y}$ mice spent more time interacting with the familiar mice (mean, $178.05 \mathrm{~s} ; \mathrm{SD}, 63.02)$ than the control mice did (no transgene: mean, $86.58 \mathrm{~s}$; SD, 47.92; Chat Cre: mean, $82.38 \mathrm{~s}$; SD, 51.89; Mecp2 flox: mean, 111.31 s; SD, 43.97). These data are consistent with the idea that selective deletion of Mecp2 from cholinergic neurons alters the ability of the animals to distinguish between novel and familiar stimuli but did not affect social interactions per se. Testing on a comprehensive behavioral battery revealed no other behavioral deficits (Table 2).

\section{Electrophysiological recording of the PRH}

Recognition memory is thought to engage circuits including the $\mathrm{PRH}$, which receives cholinergic projections from neurons in the basal forebrain. Given that Chat-Mecp2 $2^{-/ y}$ mice are selectively impaired in assays of recognition mem- 
Table 2: Chat-Mecp2 ${ }^{-/ y}$ Mice have reduced survival but are not impaired on other behavioral phenotypes

\begin{tabular}{lll}
\hline \multicolumn{1}{c}{ Test } & Phenotype tested & Phenotype present in Chat-Mecp2 $2^{-/ y}$ mice ? \\
General health exam & Weight & NS from no transgene; Mecp2 flox \\
General health exam & Stereotypies & No \\
Elevated plus maze & Anxiety-like behavior & No \\
Light/dark box & Anxiety-like behavior & No \\
Open field arena & Anxiety-like behavior & No \\
Open field arena & Hypo/hyperactivity & No \\
Rotarod & Motor learning & NS from no transgene; Mecp2 flox \\
Rotarod & Motor coordination & NS from no transgene; Mecp2 flox \\
Grip strength meter & Motor strength & No \\
Prepulse inhibition & Sensory gating & No \\
Passive avoidance & Contextual learning & No \\
Aging of animal & Reduced survival & Yes \\
\end{tabular}

ory, we next asked whether there were any overt changes in electrophysiological profile of $\mathrm{PRH}$ neurons.

In vivo extracellular recordings were collected from $\mathrm{L} 5 / 6$ of the posterior portion of the PRH (Fig. $3 A-C$ ). Although there was no significant difference in baseline firing rate (Fig. 3D; no transgene: $n=24$ units from 6 mice; Chat Cre: $n=22$ units from 11 mice; Mecp2 flox: $n=6$ units from 3 mice; Chat-Mecp2 ${ }^{-/ y}: n=20$ units from 8 mice; Kruskal-Wallis test, $\left.H_{(3)}=6.62, p=0.085\right), \mathrm{PRH}$ units from control mice (WT, Chat Cre, and Mecp2 flox mice) had highly variable firing patterns. In contrast, $\mathrm{PRH}$ units from Chat-Mecp2 $2^{-/ y}$ mice exhibited very regular and rhythmic firing (Fig. 3C). Comparison of the variability of the firing rate as measured by the Fano factor revealed a significant difference between groups (Kruskal-Wallis test, $\left.H_{(3)}=8.92, p=0.03\right)$. PRH units from Chat-Mecp2 ${ }^{-/ y}$ mice had the lowest firing rate variability (Fig. $3 E$; no transgene, mean FF $=0.612 \pm 1.250$; Chat Cre, mean FF $=0.474 \pm 0.650$; Mecp2 flox, mean FF =0.447 \pm 0.365 ; Chat-Mecp2 $2^{-/ y}$, mean FF $=0.248 \pm 0.431$ ).

\section{Optogenetic stimulation of cholinergic input to the PRH}

The above results indicate that Mecp2 deletion from cholinergic neurons has an important functional effect on $\mathrm{PRH}$ firing at baseline. We next asked whether Mecp2 deletion from cholinergic neurons affected the response of the $\mathrm{PRH}$ to the stimulation of endogenous acetylcholine release. The $\mathrm{PRH}$ receives the majority of its cholinergic innervation from neurons in the NBM (Woolf, 1991; Kondo and Zaborszky, 2016). To acutely stimulate acetylcholine release in the $\mathrm{PRH}$, we infected Chat Cre and Chat$\mathrm{Mecp2}^{-/ y}$ mice with a Cre-dependent AAV (adenoassociated virus) expressing the channelrhodopsin variants oChIEF or ChETA fused to tdTomato (Fig. $4 A, B$ ). Cholinergic neurons in the NBM of both Chat Cre and Chat-Mecp2 $2^{-/ y}$ mice expressed functional oChIEF, as indicated by fluorescent imaging of tdTomato (Fig. $4 \mathrm{C}$ ) and by optically evoked action potentials (Fig. 4D).

We next recorded $\mathrm{PRH}$ units before, during, and after laser activation of oChIEF expressed in NBM cholinergic neurons $(20 \times 1 \mathrm{~ms}$ pulses at $10 \mathrm{~Hz})$. In control animals, the stimulation of NBM cholinergic neurons changed the variance of the interspike intervals (Fig. $5 A$ ) as measured by the $F$ statistic ( variance $_{\text {pre }}$ /variance post $_{\text {). }}$ PRH units in
Chat-Mecp2 $2^{-/ y}$ did not respond to optical stimulation of cholinergic input; there was no change in ISI variance (Fig. $5 B$ ) before versus after optical stimulation of cholinergic input.

Figure $5 \mathrm{C}$ shows a heat map of response for each $\mathrm{PRH}$ unit as a function of time before and after optical stimulation of cholinergic input for control mice (Fig. 5C, left) and Chat-Mecp2 ${ }^{-1 y}$ mice (Fig. $5 C$, right). While $22.7 \%$ of control PRH units (5 of 22) exhibited a change in firing rate variability in the first $140 \mathrm{~s}$ time period following laser stimulation, there were no immediate laser stimulationassociated responses detected in Chat-Mecp2-ly $\mathrm{PRH}$ units. The proportion of units exhibiting delayed responses to laser stimulation was similar between control and Chat-Mecp2-/y mice (Chat Cre mice: $36.4 \%, 8$ of 22; Chat-Mecp2 $2^{-/ y}: 35 \%, 7$ of 20). In contrast, the proportion of units with no detectable response to optical stimulation was higher in Chat-Mecp2 $2^{-/ y}$ mice $(65 \%, 13$ of 20$)$ than in controls $(40.9 \%, 9$ of 22$)$. These distributions of responses were statistically significantly different (Fig. 6D; $\chi^{2}$ test for homogeneity, $\mathrm{CR}(2)=6.02, p=0.049$ ), which is consistent with a general loss of ACh modulation of $\mathrm{PRH}$ activity in the Chat-Mecp2 $/ y$ mice.

\section{Effect of donepezil on Chat-Mecp2-/y phenotype}

Mecp2 deletion from cholinergic neurons impaired recognition memory performance and altered $\mathrm{PRH}$ cell firing at baseline and after the stimulation of cholinergic projection neurons. If these deficits resulted from impaired cholinergic signaling per se, then we predicted that these phenotypes would be reversed by pharmacological inhibition of acetylcholine degradation by acetylcholinesterase (AChE).

Chronic administration of the AChE inhibitor donepezil ( $0.3 \mathrm{mg} / \mathrm{ml} / \mathrm{d}$, delivered via a subcutaneous minipump) rescued the performance of Chat-Mecp2-1y mice in the novel object recognition task (Fig. 6A). Chat-Mecp2-/y mice treated with donepezil (Chat-Mecp2 ${ }^{-1 y}+\mathrm{Dpz}$ ) spent significantly more time exploring a novel object than a familiar object (Fig. 6A; Chat-Mecp2 $2^{-1 y}+$ saline mice, $n=$ 8; Chat-Mecp2 $2^{-/ y}+$ Dpz mice, $n=10$ ). In fact, their behavior was qualitatively similar to that of control mice, which is consistent with the idea that donepezil blockade of AChE activity was sufficient to rescue the ability of the mice to distinguish between novel and familiar stimuli. 

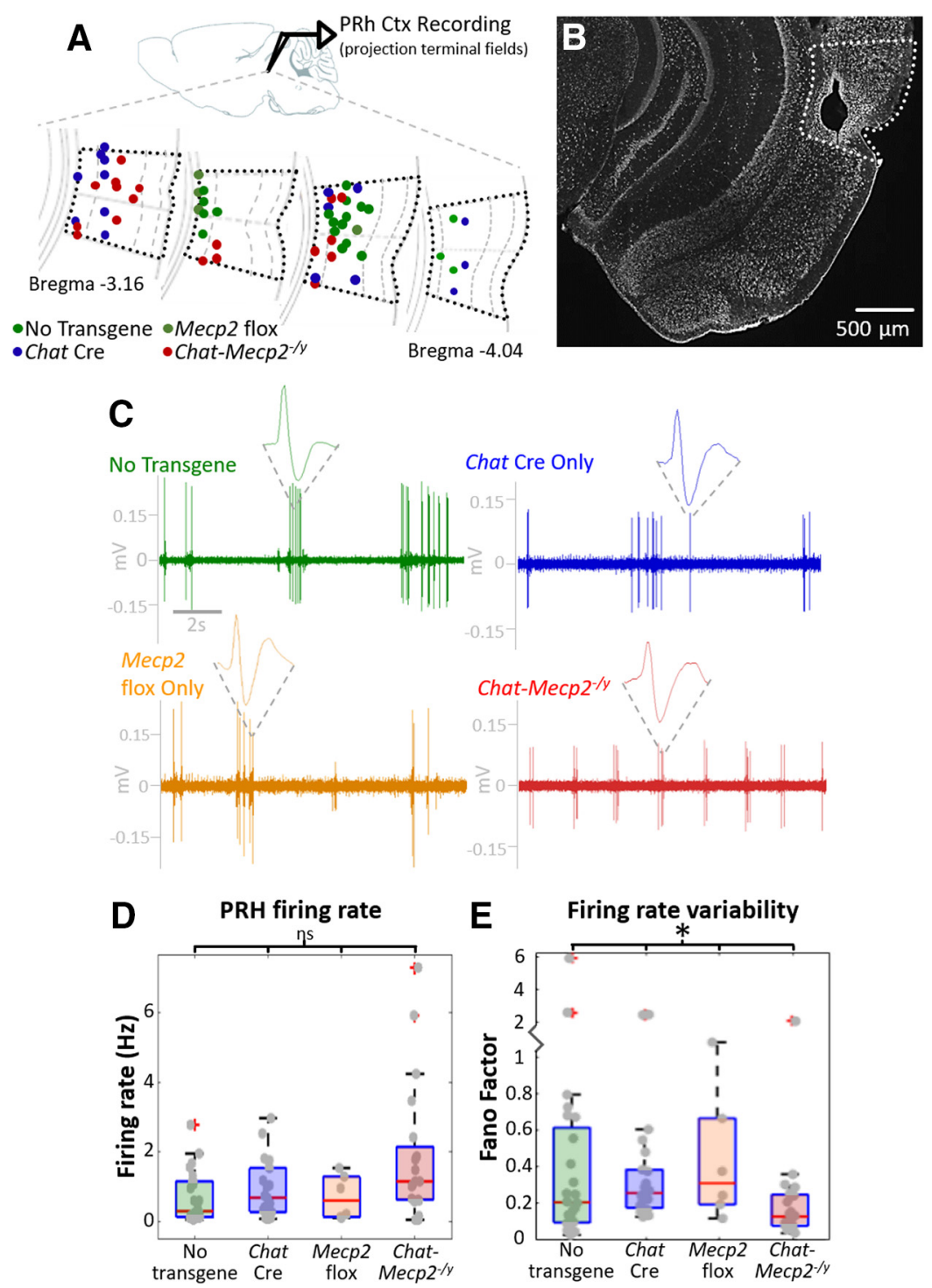

Figure 3. Neuronal firing in the $\mathrm{PRH}$ is highly variable, and this variability is lost in Chat-Mecp2-ly mice. $\boldsymbol{A}$, In vivo recordings were collected from layers 5 and 6 of the PRH. B, Sample Nissl staining and electrolytic lesion marking recording sites in the PRH (white dotted line). $\boldsymbol{C}$, Representative recordings show the highly variable baseline firing in controls that is lost in Chat-Mecp2-/y mice. $\boldsymbol{D}$, There was no difference between genotypes in baseline firing rates in the PRH (Kruskal-Wallis test: $H_{(3)}=6.62 ; p=0.085$ ). $\boldsymbol{E}$, Variability of firing rate as measured by the Fano factor was significantly different between groups (Kruskal-Wallis test: $H_{(3)}=8.92$; $p=0.03$ ). Chat-Mecp2 $2^{-/ y}$ mice had a lower firing rate variability than all three controls (no transgene: mean FF $=0.612 \pm 1.250 ;$ Chat Cre: mean FF $=0.474 \pm 0.650 ;$ Mecp2 flox: mean FF $=0.447 \pm 0.365$; Chat-Mecp2 $2^{-1 y}$ : mean FF $=0.248 \pm 0.431$ ). No transgene: $n=24$ units from 6 mice; Chat Cre: $n=22$ units from 11 mice; Mecp2 flox: $n=6$ units from 3 mice; Chat-Mecp2 $2^{-/ y}: n=20$ units from 8 mice. $* p \leq 0.05$, ns $=$ non significant.

We next tested whether the electrophysiological effects of cholinergic Mecp2 deletion could similarly be rescued by donepezil treatment. Figure $6 B$ (top) shows a representative extracellular record obtained from a PRH L5/6 neuron in an anesthetized Chat-Mecp2 ${ }^{-/ y}$ mouse treated with donepezil. Units recorded in Chat-Mecp2 ${ }^{-/ y}+\mathrm{Dpz}$ mice had highly variable firing patterns reminiscent of the electrophysiological profiles of control mice (Fig. 5B, bottom).

We also tested the effect of donepezil treatment on the $\mathrm{PRH}$ response to the stimulation of cholinergic input. Figure $6 \mathrm{C}$ shows a representative $\mathrm{PRH}$ unit from a Chat-
$\mathrm{Mecp}^{-1 y}+\mathrm{Dpz}$ mouse in which the cholinergic input to the PRH has been optically stimulated. The box plot of ISIs (Fig. 6C, left) obtained before and after stimulation shows a change in the variance of ISI. Figure $6 D$ shows a heat map of responses of Chat-Mecp2-ly $+\mathrm{DPz} \mathrm{PRH}$ units as a function of time since laser stimulation. The early laser response rate of Chat-Mecp2 ${ }^{-/ y}+\mathrm{DPz} \mathrm{PRH}$ units is partially rescued when compared with untreated Chat-Mecp2 $2^{-/ y}$, increasing from a value of 0 to $16.7 \%$ (2 of $12 \mathrm{PRH}$ units). The proportion of units with delayed responses was slightly higher than in control or ChatMecp2 $^{-/ y}$ mice (Chat-Mecp2-/y + Dpz: $41.7 \%, 5$ of $12 \mathrm{PRH}$ 
A

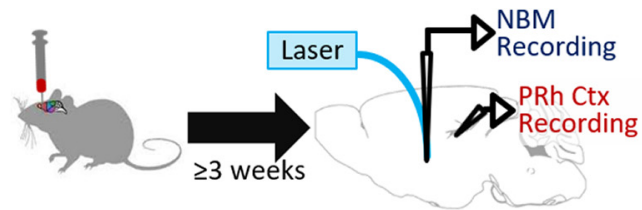

Viral Injection into NBM

In vivo electrophysiology
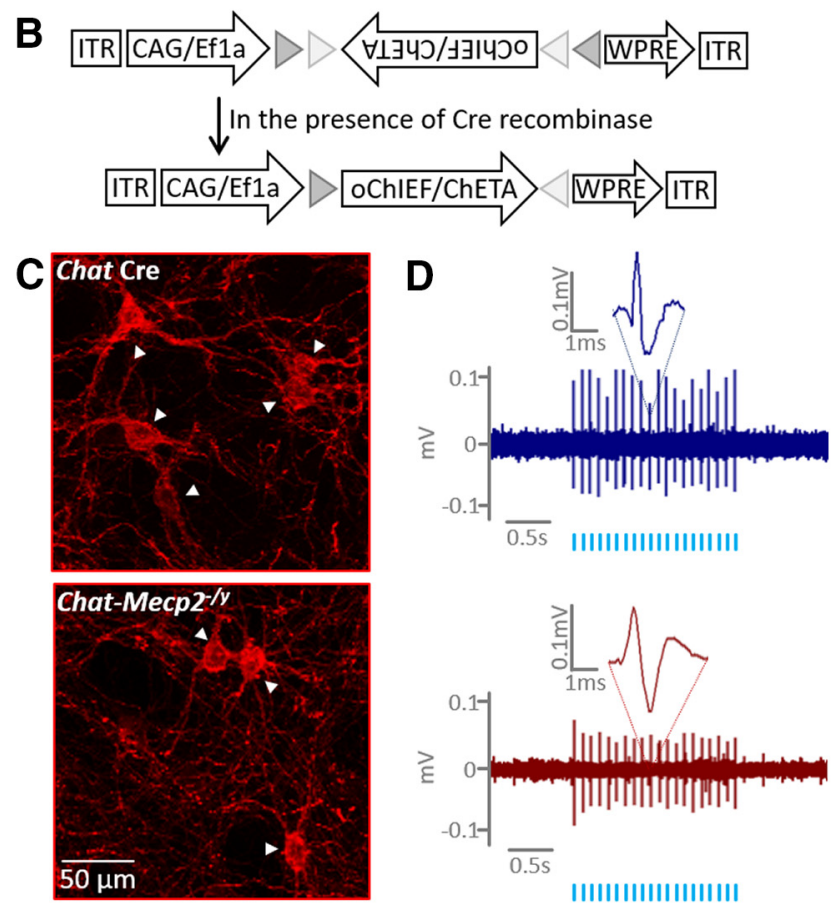

Figure 4. Strategy for optogenetic stimulation of cholinergic neurons. $\boldsymbol{A}$, Schematic of the experimental paradigm. $\boldsymbol{B}$, A viral vector encoding an optically activated excitatory ion channel is injected into the NBM. The viral vector is of a flip excision switch design such that it will be expressed only in the presence of Cre recombinase. $\boldsymbol{C}$, Representative images of virally labeled cholinergic neurons (white arrowheads) from a control mouse (blue, top) and a Chat-Mecp2-/y mouse (red, bottom). $\boldsymbol{D}$, Representative optically evoked action potentials in the NBM of a control mouse (top) and a Chat-Mecp2 ${ }^{-/ y}$ mouse (bottom). The timing of laser pulses delivered into the NBM is indicated by light blue hash marks.

units), while the proportion of units with no detectable response was similar to the control levels of $\sim 40 \%$ $(41.7 \%, 5$ of $12 \mathrm{PRH}$ units). However, the difference in distribution of response types between Chat-Mecp2-1y and Chat-Mecp2 $2^{-1 y}+$ Dpz mice did not reach statistical significance (Fig. 6E; $\chi^{2}$ test for homogeneity, $\mathrm{CR}(2)=$ $4.15, p=0.126)$.

\section{Discussion}

In this study, we assessed the behavioral and electrophysiological consequences of selective Mecp2 deletion from cholinergic neurons. Mice with Mecp2 deletion from cholinergic neurons were selectively impaired in assays of recognition memory. Because of the prominent role of the $\mathrm{PRH}$ in recognition memory, we focused our electrophysiological analyses on $\mathrm{PRH}$, where we found subtle alterations in baseline firing of L5/6 neurons in mice with selective knockout of Mecp2 from cholinergic neurons (Dere et al., 2007). Selective deletion of Mecp2 from cholinergic neurons also ablated responses to the stimulation of cholinergic input to $\mathrm{PRH}$. Finally, we demonstrated that both the behavioral and the electrophysiological profiles of cholinergic Mecp2 deletion were rescued by inhibiting ACh breakdown with donepezil.

Chat-Mecp2 ${ }^{-/ y}$ mice were selectively impaired in recognition memory tasks-both in tests of novel versus familiar object and in novel versus familiar conspecific recognition. This is consistent with the results of the study by Zhang et al. (2016), who showed that Chat-Mecp2-/y mice were impaired on the recognition of a familiar conspecific, although they did not assay novel object recognition. The selective nature of the observed cognitive deficit is an intriguing result as cholinergic signaling is known to be vital for performance on both spatial and emotional memory tasks, such as the Morris water maze and the cue-conditioned and/or context-conditioned fear assays (McNamara and Skelton, 1993; Gould, 2003; Jiang et al., 2016). The fact that Chat-Mecp2 $2^{-/ y}$ mice are not impaired on these tasks implies one of two possibilities. First, not all cholinergic neurons are functionally dependent on Mecp2 expression. Cholinergic neurons that project to brain areas involved in recognition memory may be functionally dependent on Mecp2 expression and are therefore functionally impaired by its deletion, while cholinergic neurons that project to areas involved in emotional and spatial memory are not. Although originally conceptualized as homogeneous, cholinergic basal forebrain neurons are actually quite diverse in terms of receptor and neurotransmitter expression and exhibit intricate topographical and functional organization that has only recently begun to be appreciated (Allen et al., 2006; Chandler and Waterhouse, 2012; Chandler et al., 2013; Saunders et al., 2015; Zaborszky et al., 2015a,b; Kondo and Zaborszky, 2016). The second possibility is that the loss of MeCP2 partially impairs cholinergic neurons and that different degrees of impairments might produce different phenotypes. Partial impairment impacts novel object recognition, but it might take more severe impairment to impact other learning phenotypes.

The fact that the deletion of Mecp2 from cholinergic neurons alters $\mathrm{PRH}$ firing both at baseline and after the stimulation of cholinergic input suggests that disruption of cholinergic signaling has several distinct effects on excitability over differing time scales. This is not surprising as $\mathrm{ACh}$ is thought to exert its effects via both tonic and transient signaling mechanisms (for review, see Ballinger et al., 2016). The degree to which these different modes of signaling contribute to different or overlapping cognitive functions is not well understood. Our observation that deleting Mecp2 in cholinergic neurons affects both the baseline rate and pattern of firing of L5/6 $\mathrm{PRH}$ neurons as well as the response to stimulation of cholinergic input supports the idea that cholinergic transmission via both of these signaling mechanisms may be involved in the synaptic regulation of $\mathrm{PRH}$ neuron excitability. 


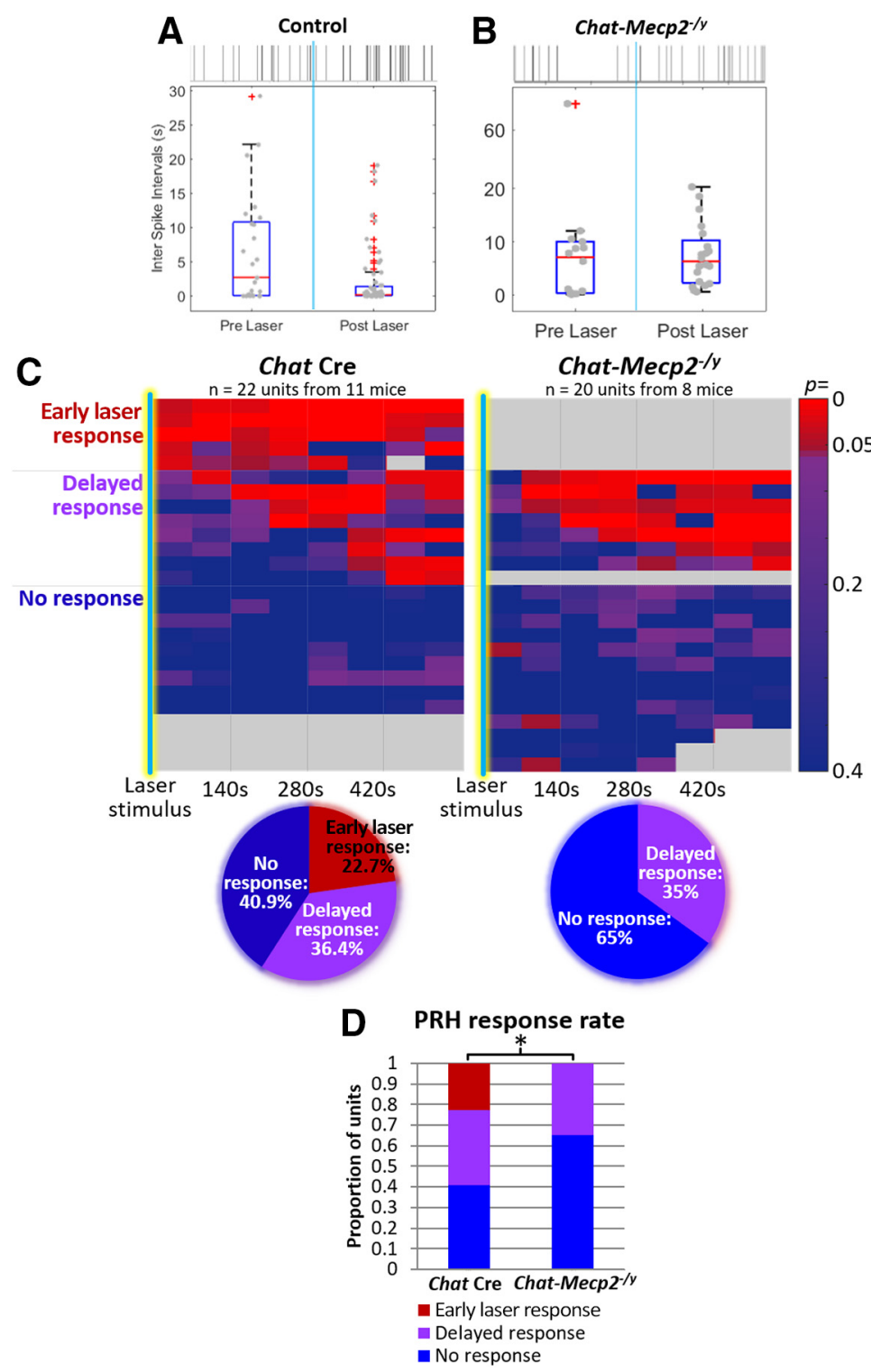

Figure 5. $\mathrm{PRH}$ response to the stimulation of endogenous cholinergic signaling is impaired in Chat-Mecp $2^{-/ y}$ mice. $\boldsymbol{A}, \boldsymbol{B}$, Representative data from a PRH unit in a control mouse exhibiting a response to stimulation of cholinergic input $(\boldsymbol{A})$ and a $\mathrm{PRH}$ unit from an Chat-Mecp2 $2^{-/ y}$ mouse (B). Top, Representative raster plot of spikes. Vertical light blue bar indicates timing of optical stimulation. Bottom, Box plot of interspike intervals. $\boldsymbol{C}$, Heat map of $p$ values as a function of time since optical stimulation for PRH units from control mice (left) and from Chat-Mecp2-/y mice (right). Responses either occurred in the first time period following laser stimulation or were delayed. Each row represents a separate unit. The results are summarized in pie charts at bottom. $\boldsymbol{D}$, Summary of differing response rates between control and Chat-Mecp2 $2^{-/ y}$ units $\left(\chi^{2}\right.$ test for homogeneity: $\left.\mathrm{CR}(2)=6.02 ; p=0.049\right)$. $* p \leq 0.05$.

At baseline, the effect of impairing cholinergic signaling via Mecp2 deletion is to reduce the variability of firing. This may represent a loss of dynamic range over which individual neurons can encode. The function of ongoing, tonic ACh release in the $\mathrm{PRH}$ may therefore be to increase this dynamic range. In addition, the effect of Chat$\mathrm{Mecp}^{-/ y}$ on the response of PRH neurons to optogenetic stimulation of cholinergic input is to ablate any changes in firing of the target $\mathrm{PRH}$ neurons. This may represent reduced functional connectivity of the NBM-PRH circuit. This loss of functional connectivity and dynamic range may impair novel object recognition encoding and therefore underlie the behavioral impairment. As our recordings have all been collected from anesthetized mice, a critical next step is to record from awake animals during behavior performance to clarify the relationship between these electrophysiological phenotypes and behavioral impairment. Furthermore, the current experiments have used cholinergic cell body stimulation to investigate the integrity of the NBM-PRH circuit. However, cholinergic NBM neurons are known to project broadly to a variety of brain areas (see Wu et al., 2014), and it is therefore impossible to determine whether the effects shown here are specific to the PRH or are downstream effects from other cholinergically innervated areas. In fact, when Mecp2 is reexpressed in cholinergic neurons in Mecp2 knock-out 

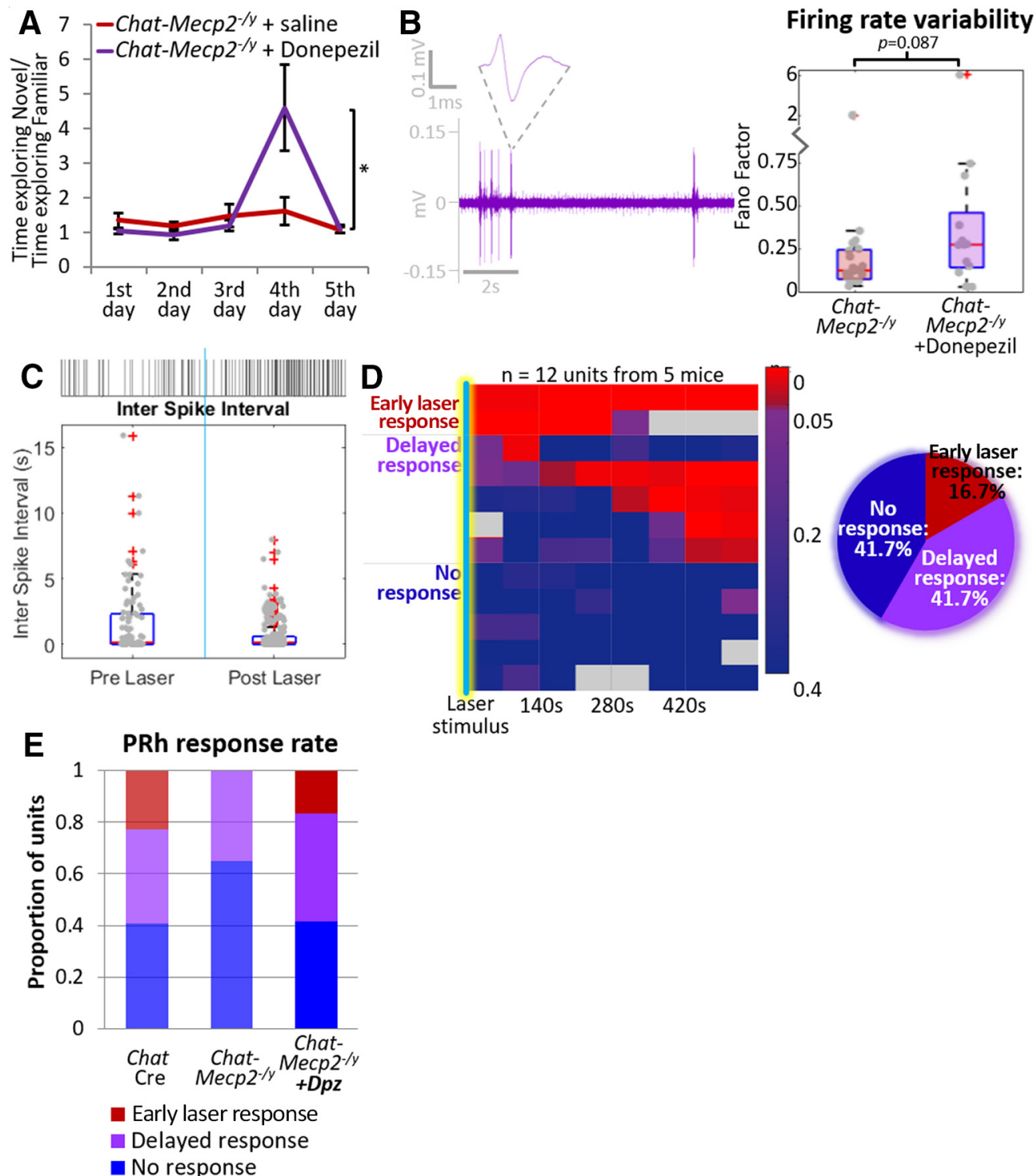

Figure 6. Donepezil treatment of Chat-Mecp2-ly mice rescues both behavioral and electrophysiological impairments. $\boldsymbol{A}$, Chronic treatment with systemic donepezil, a drug that inhibits acetylcholinesterase, administered subcutaneously for 2 weeks rescued behavioral impairment (Chat-Mecp2 $2^{-l y}+$ saline, $n=8$; Chat-Mecp2 $2^{-l y}+\mathrm{Dpz}, n=10$; Wilcoxon rank sum test: rank sum $=120 ; p$ $=0.03)$. $\boldsymbol{B}$, Representative raw data trace showing that baseline firing variability was rescued on treatment with donepezil. Firing rate variability was increased in Chat-Mecp2 ${ }^{-/ y}$ mice after treatment with donepezil (Chat-Mecp2 ${ }^{-/ y}$, mean $\mathrm{FF}=0.248 \pm 0.431$; Chat-Mecp2 $2^{-/ y}+\mathrm{Dpz}$, mean FF $=0.735 \pm 1.620$ ), although this difference did not reach statistical significance (Wilcoxon rank sum test: rank sum $=293, p=0.087)$. $\boldsymbol{C}$, Sample response to optogenetic stimulation in a Chat-Mecp2-/y mouse after treatment with donepezil. At top is shown a raster plot of action potentials before and after stimulation of cholinergic input (indicated by light blue vertical bar). At bottom is shown a box plot of interspike intervals obtained before and after optical stimulation of cholinergic neurons in the NBM. $\boldsymbol{D}$, Heat map of $p$ values as a function of time since optical stimulation. Responses either occurred in the first time period following laser stimulation or were delayed and are summarized in the pie chart at bottom. $\boldsymbol{E}$, Summary of response types. Early laser responses were restored in Chat-Mecp $2^{-l y}$ mice treated with donepezil. ( $\chi^{2}$ test for homogeneity: $\left.\operatorname{CR}(2)=4.15 ; p=0.126\right)$. $* p \leq 0.05$.

mice, the deficit in recognition of a familiar conspecific persists, suggesting that, although the present results implicate cholinergic Mecp2 expression as necessary for intact recognition memory, it is not sufficient (Zhou et al., 2017). Thus, there are likely other relevant brain areas through which the cholinergic basal forebrain exerts its effect on recognition memory performance. Future anal$y$ sis of the functional connectivity of other brain areas in the context of cholinergic Mecp2 deletion may clarify their contribution to the behavioral phenotype documented here. Further work investigating cholinergic input to the $\mathrm{PRH}$ in Chat-Mecp2 $2^{-/ y}$ mice using terminal field optogenetic stimulation and neurotransmitter release measurements is also needed to clarify the role of cholinergic signaling in this brain area and the effects of Mecp2 disruption on it.

It is possible that the electrophysiological and behavioral deficits discussed above are a consequence of a 
"sick neuron" syndrome induced by the catastrophic effects of Mecp2 deletion in a subpopulation of neurons or that these effects are mediated by a neurotransmitter other than ACh, as cholinergic neurons are known to synthesize many different neurotransmitters (Tkatch et al., 1998; Allen et al., 2006; Saunders et al., 2015). If these deficits are truly due to a cholinergic signaling impairment, we would expect that they might be rescued by the inhibition of ACh breakdown and boosting of the cholinergic signal. This was indeed the case: the fact that the inhibition of ACh breakdown with donepezil rescued these impairments demonstrates an essential role of ACh per se in mediating the phenotype. The donepezil rescue of behavior was seemingly more robust than the donepezil rescue of the electrophysiological phenotype; however, it is difficult to equate magnitudes across such very different methods.

In conclusion, Mecp2 deletion from cholinergic neurons leads to selective impairment of recognition memory, reduced variability of $\mathrm{PRH}$ firing, and reduced $\mathrm{PRH}$ responsivity to the stimulation of cholinergic input. Cholinergic signaling is therefore an important mediator of cognitive deficits in mice lacking MeCP2, and Mecp2 expression is vital for cholinergic mediation of recognition memory.

\section{References}

Allen TG, Abogadie FC, Brown DA (2006) Simultaneous release of glutamate and acetylcholine from single magnocellular "cholinergic" basal forebrain neurons. J Neurosci 26:1588-1595.

Amir RE, Van den Veyver IB, Wan M, Tran CQ, Francke U, Zoghbi HY (1999) Rett syndrome is caused by mutations in X-linked MECP2, encoding methyl-CpG-binding protein 2. Nat Genet 23:185-188.

Ballinger EC, Ananth M, Talmage DA, Role LW (2016) Basal forebrain cholinergic circuits and signaling in cognition and cognitive decline. Neuron 91:1199-1218.

Beaudin SA, Singh T, Agster KL, Burwell RD (2013) Borders and comparative cytoarchitecture of the perirhinal and postrhinal cortices in an $\mathrm{F} 1$ hybrid mouse. Cereb Cortex 23:460-476.

Brašić JR, Bibat G, Kumar A, Zhou Y, Hilton J, Yablonski ME, Dogan AS, Guevara MR, Stephane M, Johnston M, Wong DF, Naidu S (2012) Correlation of the vesicular acetylcholine transporter densities in the striata to the clinical abilities of women with rett syndrome. Synapse 66:471-482.

Chahrour M, Jung SY, Shaw C, Zhou X, Wong STC, Qin J, Zoghbi HY (2008) MeCP2, a key contributor to neurological disease, activates and represses transcription. Science 320:1224-1229.

Chandler D, Waterhouse BD (2012) Evidence for broad versus segregated projections from cholinergic and noradrenergic nuclei to functionally and anatomically discrete subregions of prefrontal cortex. Front Behav Neurosci 6:20.

Chandler DJ, Lamperski CS, Waterhouse BD (2013) Identification and distribution of projections from monoaminergic and cholinergic nuclei to functionally differentiated subregions of prefrontal cortex. Brain Res 1522:38-58.

Chao HT, Chen H, Samaco RC, Xue M, Chahrour M, Yoo J, Neul JL, Gong S, Lu HC, Heintz N, Ekker M, Rubenstein JL, Noebels JL, Rosenmund C, Zoghbi HY (2010) Dysfunction in GABA signalling mediates autism-like stereotypies and Rett syndrome phenotypes. Nature 468:263-269.

Dere E, Huston JP, De Souza Silva MA (2007) The pharmacology, neuroanatomy and neurogenetics of one-trial object recognition in rodents. Neurosci Biobehav Rev 31:673-704.

Fyffe SL, Neul JL, Samaco RC, Chao HT, Ben-Shachar S, Moretti P, McGill BE, Goulding EH, Sullivan E, Tecott LH, Zoghbi HY (2008) Deletion of Mecp2 in Sim1-expressing neurons reveals a critical role for MeCP2 in feeding behavior, aggression, and the response to stress. Neuron 59:947-958.

Gould TJ (2003) Nicotine produces a within-subject enhancement of contextual fear conditioning in C57BL/6 mice independent of sex. Integr Physiol Behav Sci 38:124-132.

Gray CM, Maldonado PE, Wilson M, McNaughton B (1995) Tetrodes markedly improve the reliability and yield of multiple single-unit isolation from multi-unit recordings in cat striate cortex. J Neurosci Methods 63:43-54.

Hagberg B, Aicardi J, Dias K, Ramos O (1983) A progressive syndrome of autism, dementia, ataxia, and loss of purposeful hand use in girls: Rett's syndrome: report of 35 cases. Ann Neurol 14:471-479.

Ito-Ishida A, Ure K, Chen H, Swann JW, Zoghbi HY (2015) Loss of MeCP2 in parvalbumin-and somatostatin-expressing neurons in mice leads to distinct Rett syndrome-like phenotypes. Neuron 88:651-658.

Jiang L, Kundu S, Lederman JD, López-Hernández GY, Ballinger EC Wang S, Talmage DA, Role LW (2016) Cholinergic signaling controls conditioned fear behaviors and enhances plasticity of cortical-amygdala circuits. Neuron 90:1057-1070.

Kitt C, Troncoso J, Price D, Naidu S, Moser H (1990) Pathological changes in substantia nigra and basal forebrain neurons in Rett syndrome. Ann Neurol 28:416.

Kondo H, Zaborszky L (2016) Topographic organization of the basa forebrain projections to the perirhinal, postrhinal, and entorhinal cortex in rats. J Comp Neurol 524:2503-2515.

Lewis JD, Meehan RR, Henzel WJ, Maurer-Fogy I, Jeppesen P, Klein $F$, Bird A (1992) Purification, sequence, and cellular localization of a novel chromosomal protein that binds to methylated DNA. Cell 69:905-914.

McNamara RK, Skelton RW (1993) The neuropharmacological and neurochemical basis of place learning in the Morris water maze. Brain Res Rev 18:33-49.

Meng X, Wang W, Lu H, He LJ, Chen W, Chao ES, Fiorotto ML, Tang B, Herrera JA, Seymour ML, Neul JL, Pereira FA, Tang J, Xue M, Zoghbi HY (2016) Manipulations of MeCP2 in glutamatergic neurons highlight their contributions to Rett and other neurological disorders. Elife 5:e14199.

Moretti P, Bouwknecht JA, Teague R, Paylor R, Zoghbi HY (2005) Abnormalities of social interactions and home-cage behavior in a mouse model of Rett syndrome. Hum Mol Genet 14:205-220.

Neul JL, Kaufmann WE, Glaze DG, Christodoulou J, Clarke AJ, Bahi-Buisson N, Leonard H, Bailey ME, Schanen NC, Zappella M, Renieri A, Huppke P, Percy AK (2010) Rett syndrome: revised diagnostic criteria and nomenclature. Ann Neurol 68:944-950.

Oginsky MF, Cui N, Zhong W, Johnson CM, Jiang C (2014) Alterations in the cholinergic system of brain stem neurons in a mouse model of Rett syndrome. Am J Physiol Cell Physiol 307:C508C520.

Rett A (1966) On an until now unknown disease of a congenital metabolic disorder. Krankenschwester 19:121-122.

Ricceri L, De Filippis B, Fuso A, Laviola G (2011) Cholinergic hypofunction in MeCP2-308 mice: beneficial neurobehavioural effects of neonatal choline supplementation. Behav Brain Res 221:623629.

Rudas T (1986) A Monte Carlo comparison of the small sample behaviour of the Pearson, the likelihood ratio and the CressieRead statistics. J Stat Comput Simul 24:107-120.

Samaco RC, Mandel-Brehm C, Chao HT, Ward CS, Fyffe-Maricich SL, Ren J, Hyland K, Thaller C, Maricich SM, Humphreys P, Greer JJ, Percy A, Glaze DG, Zoghbi HY, Neul JL (2009) Loss of MeCP2 in aminergic neurons causes cell-autonomous defects in neurotransmitter synthesis and specific behavioral abnormalities. Proc Natl Acad Sci U S A 106:21966-21971.

Saunders A, Granger AJ, Sabatini BL (2015) Corelease of acetylcholine and GABA from cholinergic forebrain neurons. Elife 4:e06412.

Takeuchi H, Iba M, Inoue H, Higuchi M, Takao K, Tsukita K, Karatsu Y, Iwamoto Y, Miyakawa T, Suhara T, Trojanowski JQ, Lee VM, Takahashi R (2011) P301S mutant human tau transgenic mice 
manifest early symptoms of human tauopathies with dementia and altered sensorimotor gating. PLoS One 6:e21050.

Thorvaldsen S, Flå T, Willassen NP (2010) DeltaProt: a software toolbox for comparative genomics. BMC Bioinformatics 11:573.

Tkatch T, Baranauskas G, Surmeier DJ (1998) Basal forebrain neurons adjacent to the globus pallidus co-express GABAergic and cholinergic marker mRNAs. Neuroreport 9:1935-1939.

Ward BC, Kolodny NH, Nag N, Berger-Sweeney JE (2009) Neurochemical changes in a mouse model of Rett syndrome: changes over time and in response to perinatal choline nutritional supplementation. J Neurochem 108:361-371.

Wenk GL (1997) Rett syndrome: neurobiological changes underlying specific symptoms. Prog Neurobiol 51:383-391.

Wenk GL, Mobley SL (1996) Choline acetyltransferase activity and vesamicol binding in rett syndrome and in Rats with nucleus basalis lesions. Neuroscience 73:79-84.

Wenk GL, Hauss-Wegrzyniak B (1999) Altered cholinergic function in the basal forebrain of girls with Rett syndrome. Neuropediatrics 30:125-129.

Woolf NJ (1991) Cholinergic systems in mammalian brain and spinal cord. Prog Neurobiol 37:475-524.

Wu H, Williams J, Nathans J (2014) Complete morphologies of basal forebrain cholinergic neurons in the mouse. Elife 3:e02444.
Yasui DH, Scoles HA, Horike S, Meguro-Horike M, Dunaway KW, Schroeder DI, Lasalle JM (2011) 15q11.2-13.3 chromatin analysis reveals epigenetic regulation of CHRNA7 with deficiencies in Rett and autism brain. Hum Mol Genet 20:4311-4323.

Zaborszky L, Csordas A, Mosca K, Kim J, Gielow MR, Vadasz C, Nadasdy Z (2015a) Neurons in the basal forebrain project to the cortex in a complex topographic organization that reflects corticocortical connectivity patterns: an experimental study based on retrograde tracing and 3D reconstruction. Cereb Cortex 25:118137.

Zaborszky L, Duque A, Gielow M, Gombkoto P, Nadasdy Z, Somogyi $\mathrm{J}(2015 \mathrm{~b})$ Organization of the basal forebrain cholinergic projection system: specific or diffuse? In: The rat nervous system, Ed 4 (Paxinos G, ed), pp 491-507. Amsterdam: Elsevier.

Zhang Y, Cao SX, Sun P, He HY, Yang CH, Chen XJ, Shen CJ, Wang XD, Chen Z, Berg DK, Duan S, Li XM (2016) Loss of MeCP2 in cholinergic neurons causes part of RTT-like phenotypes via $\alpha 7$ receptor in hippocampus. Cell Res 26:728-742.

Zhou H, Wu W, Zhang Y, He H, Yuan Z, Zhu Z, Zhao Z (2017) Selective preservation of cholinergic MeCP2 rescues specific Rett-syndrome-like phenotypes in MeCP2stop mice. Behav Brain Res 322:51-59. 